Research Paper

\title{
Association of BTBD7 with Metastasis and Poor Prognosis in Non-Small-Cell Lung Cancer Patients
}

\author{
Fan-Yan Luo ${ }^{1}$, Zi-Hou Liu ${ }^{1}$,Qin-Hua Hu${ }^{1}$, Guo-Qiang Lin ${ }^{1}{ }^{\bowtie}$, Can-E Tang ${ }^{2}{ }^{\bowtie}$,Wei-Xing Zhang1, Wei Zhuang1 \\ 1. Department of Cardiothoracic Surgery, Xiangya Hospital, Central South University; \\ 2. Institute of Medical Sciences, Xiangya Hospital, Central South University, Changsha 410008, China.
}

$\square$ Corresponding authors: Guo-Qiang Lin, Department of Cardiothoracic Surgery, Xiangya Hospital, Central South University, Changsha 410008, China. Can-E Tang, Institute of Medical Sciences, Xiangya Hospital, Central South University, Changsha 410008, China. Email: drlfy@163.com, lgq186snoopy@126.com.

(c) 2015 Ivyspring International Publisher. Reproduction is permitted for personal, noncommercial use, provided that the article is in whole, unmodified, and properly cited. See http://ivyspring.com/terms for terms and conditions.

Received: 2015.01.27; Accepted: 2015.02.09; Published: 2015.03.26

\begin{abstract}
Metastasis in lung cancer portends a poor prognosis, and the epithelial-mesenchymal transition (EMT) in lung cancer cells is considered a prerequisite to achieve metastatic potential. Recent studies indicate that BTB/POZ domain-containing protein 7 (BTBD7) regulates EMT-associated proteins in human malignancies and however, the role of BTBD7 in lung cancer have not been identified. In present study, we examined BTBD7 expression status and its association with unfavorable clinical features in non-small-cell lung cancer (NSCLC). Firstly, we studied the fresh specimens, and found that both mRNA and protein expression levels of BTBD7 in NSCLC tissue were significantly increased compared with the adjacent nontumorous lung tissue. Then, we determined BTBD7 protein expressions in the paraffin-embedded samples from NSCLC patients, and analyzed the relations of BTBD7 expression with clinicopathologic features of the patients. The results showed that incidence of metastasis in patients with positive BTBD7 expression was significantly higher than that in those with negative BTBD7 expression, and the positive BTBD7 expression rate in metastatic cases was significantly higher than that in non-metastatic ones; furthermore, Cox regression analyses revealed that BTBD7 was an independent risk factor for either metastasis or survival in NSCLC patients. Thus, we conclude that BTBD7 contributes to metastasis of NSCLC and BTBD7-positive NSCLC may have a high potential for metastasis and thereby a poor prognosis
\end{abstract}

Key words: BTB/POZ domain-containing protein 7, non-small-cell lung cancer, metastasis, prognostic factors.

\section{Introduction}

Lung cancer is one of the most common and lethal human malignancies worldwide, ranking first in terms of both incidence and mortality (1). Its prognosis remains poor despite recent advances in both diagnosis and therapy, which is attributed largely to the high incidence of distant metastasis at initial diagnosis. However, the mechanism responsible for lung cancer metastasis is still poorly defined.

$\mathrm{BTB} / \mathrm{POZ}$ domain containing protein (BTBD) is a growth suppressor protein that mainly participates in protein-protein interaction with E3 ubiquitin ligase
$(2,3)$. A recent study using MDCK (Madin-Darby canine kidney) cells showed that BTBD7, one of the spliced variants of BTBD, promotes dispersion of the cells and down-regulates E-cadherin which contributes to the metastasis inhibition in pulmonary adenocarcinoma (4-6). Moreover, BTBD7 has been proved to promote angiogenesis and metastasis of hepatocellular carcinoma (HCC) both in vitro and in vivo, but do not influence cell proliferation or colony formation (7). However, whether BTBD7 participates in the metastasis and affects the prognosis of 
non-small-cell lung cancer (NSCLC) requires to be clarified. Here, we determined the BTBD7 expression status in NSCLC and its potential clinical significance.

\section{Materials and Methods}

All procedures of this study were approved by the Xiangya Hospital Ethics Committee.

\section{Fresh tissue samples}

Matched fresh specimens of NSCLC and adjacent nontumorous lung tissues collected from 20 patients were quickly frozen and kept at $-70^{\circ} \mathrm{C}$ for quantitative reverse-transcriptase polymerase chain reaction (qRT-PCR) and Western blot analysis.

\section{Paraffin-embedded tissue samples}

The paraffin-embedded tumor tissue samples were obtained from the follow-up database of primary lung cancer patients undergoing surgical treatment in our hospital between 2002 and 2011. Thirty-five NSCLC patients with definite evidence of metastases were enrolled (metastatic group), and the involved distant organs included brain, liver, bone, contralateral lung and so on; another 58 NSCLC patients with no evidence of metastasis over 2-year follow-up period were selected as control (non-metastatic group). In metastatic group, 24 cases were male and 11 cases were female with mean age (60.43 \pm 6.80$)$ years; 20 cases were squamous cell carcinoma, and 15 were adenocarcinoma. In non-metastatic group, 48 cases were male and 10 cases were female, with mean age $(57.12 \pm 8.87)$ years; 39 cases were squamous cell carcinoma, 16 cases were adenocarcinoma and 3 cases were large cell NSCLC. There was no significant demographic difference between the two groups $(P>0.05)$.

\section{Immunohistochemical staining and scoring for BTBD7 expression}

BTBD7 immunohistochemistry was conducted on 5- $\mu \mathrm{m}$ thick, formalin-fixed, paraffin-embedded tissue sections. The slides were stained by labeled streptavidin-biotinylated peroxidase method (ZSGB-BIO, Beijing, China) according to the manufacturer's instructions. The primary antibody used was a goat ployclonal anti-BTBD7 (C-18, Santa Cruz Biotechnology, USA), and antigen retrieval was accomplished by heat treatment in a citrate buffer. Immunostaining was detected with diaminobenzidine (ZSGB-BIO, Beijing, China) and nuclei were counterstained with hematoxylin. The primary antibody was omitted in negative control samples.

The evaluation and scoring of BTBD7 expression were conducted by two independent investigators with pathological training following a blind protocol.
The immunoreactive score (IRS) described by Remmele and Stegner (8) was used to evaluate the SI (staining intensity) and PP (percentage of positive cells) of the staining as follows: IRS $=\mathrm{SI} \times \mathrm{PP}$. SI was determined as 0 , negative; 1 , weak; 2 , moderate; and 3 , strong. PP was defined as 0 , negative; $1,10 \%$ positive cells; $2,11 \%-50 \%$ positive cells; $3,51 \%-80 \%$ positive cells; and 4 , more than $80 \%$ positive cells. IRS value $\geq 3$ was considered as a positive staining result.

\section{qRT-PCR protocol}

SYBR Green fluorescent-based qRT-PCR was performed as previously described (9). Relative mRNA expression levels were calculated by the $2^{-\Delta \mathrm{Ct}}$ method, based on threshold cycle values, and were normalized to the internal control ( $\beta$-actin).

\section{Western blot analysis}

Total protein was separated by sodium dodecyl sulfate polyacrylamide gel electrophoresis and then transferred to polyvinylidene fluoride membrane (Millipore, Bedford, MA). Blocked membranes were incubated with the primary antibodies, followed by horseradish-peroxidase-conjugated secondary antibodies (KPL, Gaithersburg, MD). Bands were visualized using the enhanced chemiluminescence kit (Santa Cruz Biotechnology, Santa Cruz, CA). Target signals were quantified by BandScan software (Bio-Rad, Hercules, CA) and defined as the ratio of target protein relative to $\beta$-actin.

\section{Statistical analysis}

Statistical analysis was performed using SPSS 13.0 software (SPSS, Inc., Chicago, IL). Quantitative values are presented as mean \pm standard deviation or median (range). Student $t$ test and $\chi^{2}$ test were used for comparison of categorical and continuous variables, respectively. Kaplan-Meier model was used for survival analysis, and Cox regression model was used for survival-related multivariate analysis. Analysis of risk factors of metastasis in NSCLC was based on logistic regression model. $P<0.05$ was considered to be statistically significant.

\section{Results}

\section{Expression of BTBD7 mRNA and protein in fresh NSCLC tissue}

In the 20 matched fresh specimens, BTBD7 mRNA was significantly up-regulated in NSCLC tissue compared with adjacent nontumorous lung tissue $(0.0326 \pm 0.0065$ vs $0.0057 \pm 0.0014, P<0.01)$ (Figure 1A); the average expression level of BTBD7 protein in NSCLC tissue was significantly higher than that in adjacent nontumorous lung tissues $(1.18 \pm 0.23$ vs $0.54 \pm 0.06, P=0.017$ ) (Figure 1B). Furthermore, BTBD7 
mRNA and protein expression presented a significant positive correlation in NSCLC tissue $(r=0.698, P<0.01)$.

\section{BTBD7 protein expression in paraf- fin-embedded NSCLC tissue}

Positive immunohistochemical staining was observed mainly in the cytoplasm of the cells, and the representative immunohistochemical staining images for BTBD7-negative and -positive expression of were shown in Figure 2. In total 93 patients, 38 cases were found to be positive BTBD7 expression and 55 were negative BTBD7 expression. The overall positive rate was $40.86 \%$. In patients with positive BTBD7 expression, the incidence of metastasis was $63.53 \%$, while it was $21.82 \%$ in patients with negative BTBD7 expression; the positive BTBD7 expression rate was $65.71 \%$ $(23 / 35)$ in metastatic patients, which was $25.86 \%$ A

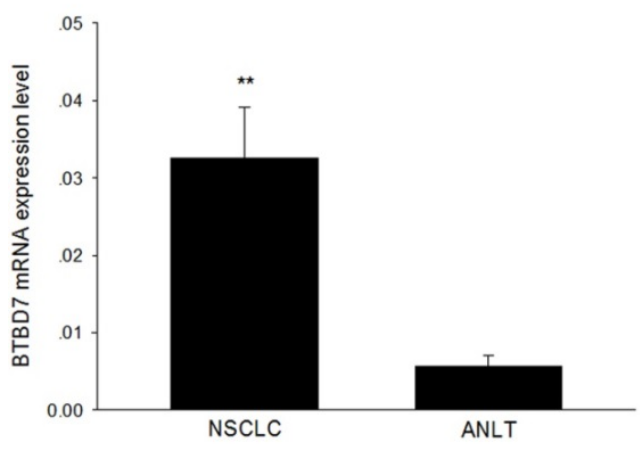

$(15 / 58)$ in non-metastatic patients, and both differences reached significance (both $P<0.05$ ).

\section{Risk factors analysis of NSCLC metastasis}

As found above, the positive BTBD7 expression rate in metastatic patients was significantly higher than that in non-metastatic ones. Logistic regression analysis was performed to further identify whether BTBD7 expression is a risk factor for metastasis in NSCLC. The results revealed that BTBD7 expression and TNM stage were independent risk factors for NSCLC metastasis and the odds ratio (OR) of developing metastasis were 10.258 in patients with positive BTBD7 expression compared with those with negative BTBD7 expression (Table 2).

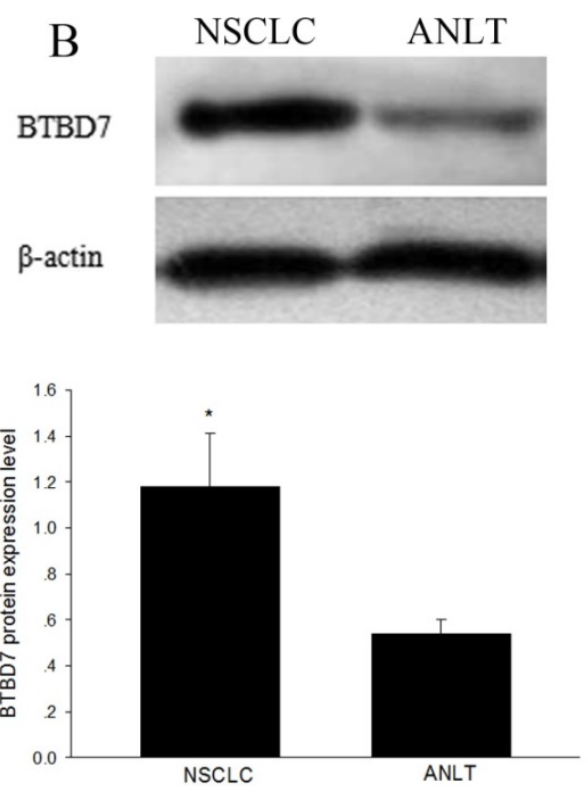

Figure 1. BTBD7 expression in 20 matched fresh tissues. (A) BTBD7 mRNA expression in NSCLC and adjacent nontumorous lung tissue (ANLT); (B) BTBD7 protein expression in NSCLC and ANLT; *( vs ANLT) $P<0.05 ; * *$ ( vs ANLT) $P<0.01$.

A

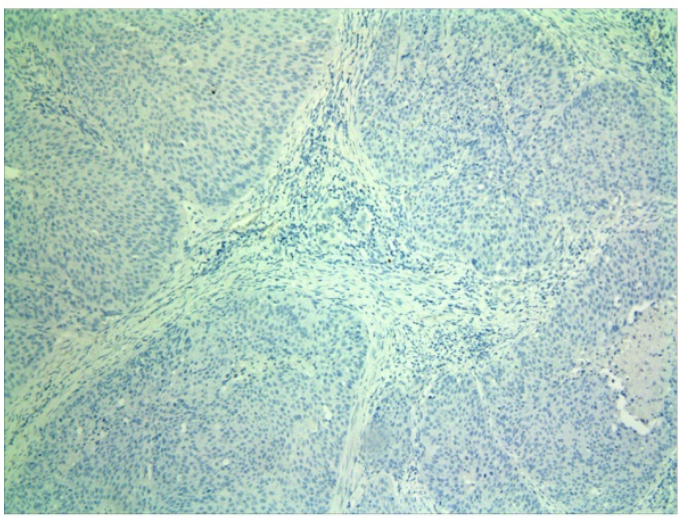

B

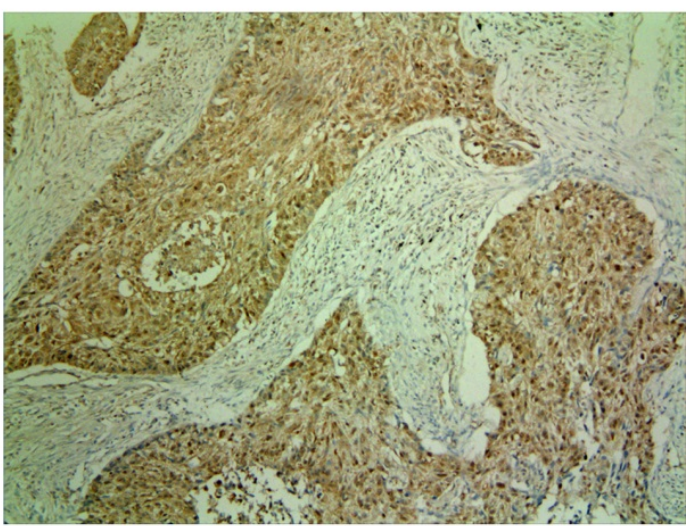

Figure 2. Immunohistochemical staining for BTBD7 expression in paraffin-embedded sections $(\times 100)$. (A) Negative BTBD7 expression; (B) Positive BTBD7 expression. 


\section{Survival analysis}

Results of Kaplan-Meier analysis showed that the median estimated survival time was $(71.6 \pm 4.7)$ months for the entire group of the 93 patients, which was $(55.2 \pm 6.2)$ months for the group of patients with positive BTBD7 expression, and was (89.2 \pm 4.8$)$ months for group of patients with negative BTBD7 expression, respectively. The difference in survival time between patients with positive and negative BTBD7 expression was statistically significant $\left(X^{2}=10.87, P<0.01\right)$, and the overall survival time curves of the two groups of patients were presented in Figure 3.

Further, the factors potentially affecting the survival of the patients were analyzed by Cox regression model. The results showed that BTBD7 expression and TNM stage were independent risk factor for survival, while age and smoking did not affect the survival of the patients. Given the marginal statistical significance, the effect of sex on the survival of the NSCLC patients' needs further investigation. The risk of short survival in patients with positive BTBD7 expression was 3.404 times greater than that in those with negative BTBD7 expression (Table 3).

\section{Discussion}

Although tremendous research efforts have been made, metastasis of lung cancer remains the major obstacle for its successful management. So it is an urgent need to find the useful biomarkers or effective therapeutic targets associated with this process. In this study, we first examined the BTBD7 expression status in NSCLC tissues, and the results showed the average expression levels of both BTBD7 mRNA and protein in NSCLC tissues were significantly higher than those in their adjacent lung tissues, which suggested that BTBD7 may be probably a biomarker for

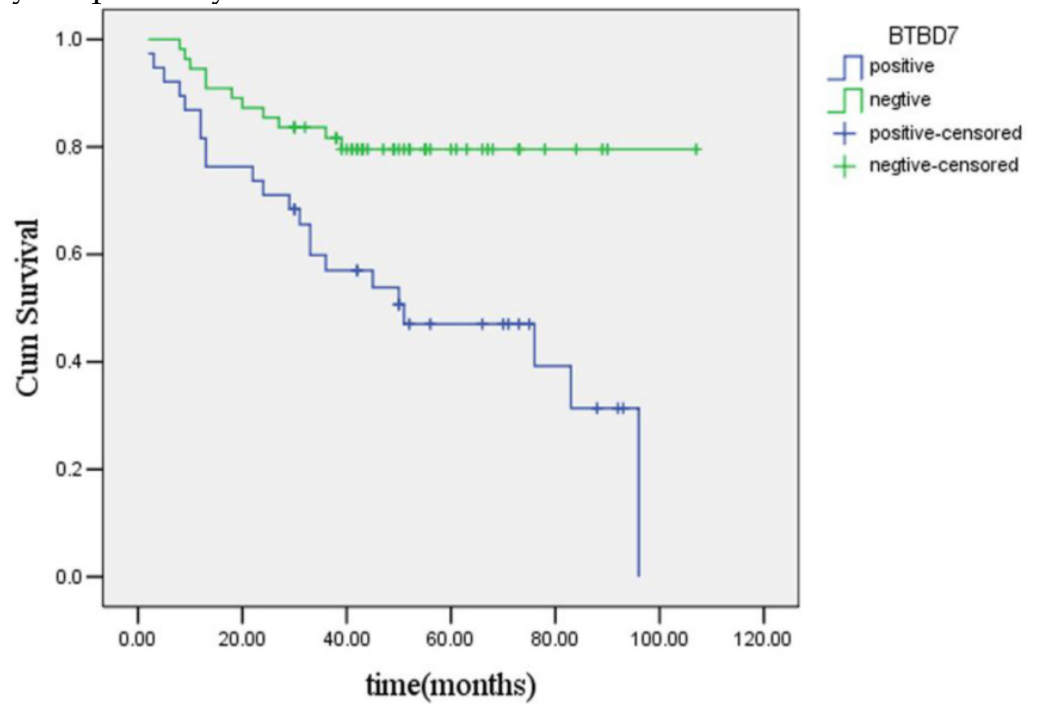

Table 1. BTBD7 expression in NSCLC patients. metastasis. confidence interval.

NSCLC. Subsequently, we determined BTBD7 protein expression in 93 NSCLC patients and however, the results showed that the overall positive expression rate of BTBD7 protein was only $40.86 \%$, so BTBD7 may not be a sensitive biomarker for NSCLC.

\begin{tabular}{llll}
\hline Variables & $n$ & \multicolumn{2}{c}{ BTBD 7 } \\
\cline { 3 - 4 } & & Positive & Negative \\
\hline Metastasis & 35 & $23(65.7 \%)$ & $12(34.3 \%)$ \\
Yes & 58 & $15(25.9 \%)$ & $43(74.1 \%)$ \\
No & & & \\
Histological type & 31 & $20(64.5 \%)$ & $11(35.5 \%)$ \\
Adenocarcinoma & 59 & $27(45.8 \%)$ & $32(54.2 \%)$ \\
Squamous cell carcinoma & 3 & $0(0.0 \%)$ & $3(100.0 \%)$ \\
Large cell carcinoma & 3 & & \\
\hline
\end{tabular}

Table 2. Logistic regression analysis of risk factors for NSCLC

\begin{tabular}{|c|c|c|c|c|c|c|}
\hline \multirow[t]{2}{*}{ Variables } & \multirow[t]{2}{*}{$B$} & \multirow[t]{2}{*}{$S E$} & \multirow[t]{2}{*}{ Sig. } & \multirow[t]{2}{*}{$O R$} & \multicolumn{2}{|c|}{$95.0 \% \mathrm{CI}$ for $\mathrm{OR}$} \\
\hline & & & & & Lower & Upper \\
\hline Age & -0.022 & 0.021 & 0.298 & 0.978 & 0.938 & 1.020 \\
\hline Sex & -0.046 & 0.837 & 0.956 & 0.955 & 0.185 & 4.922 \\
\hline Smoking & -0.083 & 0.679 & 0.357 & 0.937 & 0.269 & 3.823 \\
\hline BTBD7 & 2.328 & 0.689 & 0.001 & 10.258 & 2.658 & 39.591 \\
\hline Histological type & 0.496 & 0.633 & 0.434 & 1.642 & 0.475 & 5.677 \\
\hline TNM stage & 1.048 & 0.453 & 0.021 & 2.851 & 1.173 & 6.929 \\
\hline
\end{tabular}

$B$ coefficient for the constant; SE standard error; Sig significance; OR odds ratio; $C I$

Table 3. Cox regression analysis for survival-related factors.

\begin{tabular}{|c|c|c|c|c|c|c|}
\hline \multirow[t]{2}{*}{ Variables } & \multirow[t]{2}{*}{$B$} & \multirow[t]{2}{*}{$S E$} & \multirow[t]{2}{*}{ Sig. } & \multirow[t]{2}{*}{$H R$} & \multicolumn{2}{|c|}{$95.0 \% \mathrm{CI}$ for $H R$} \\
\hline & & & & & Lower & Upper \\
\hline Age & 0.006 & 0.023 & 0.808 & 1.006 & 0.962 & 1.051 \\
\hline Sex & -0.898 & 0.451 & 0.046 & 0.407 & 0.168 & 0.985 \\
\hline Smoking & 0.573 & 0.376 & 0.391 & 1.874 & 0.857 & 3.571 \\
\hline BTBD7 & 1.225 & 0.420 & 0.004 & 3.404 & 1.494 & 7.753 \\
\hline Histological type & 0.429 & 0.377 & 0.255 & 1.535 & 0.733 & 3.215 \\
\hline TNM stage & 1.359 & 0.247 & 0.000 & 3.891 & 2.399 & 6.310 \\
\hline
\end{tabular}


Members of the POZ gene family, characterized by a conserved BTB/POZ protein-protein interaction motif, have been implicated in human cancer $(10,11)$. BTBD7 is essential in embryonic phase of lung development because it regulates both branching morphogenesis and epithelial tissue remodeling (7). Moreover, BTBD7 has recently been demonstrated to participate in lung branching morphogenesis and epithelial tissue remodeling by regulating Snail2, fibronectin, and E-cadherin, which are associated with lung cancer invasion and metastasis (12-14). Tao and colleagues (7) found that knockdown of BTBD7, which acts as an upstream activator of epithelial-mesenchymal transition (EMT), induces E-cadherin expression, but restrains fibronectin and Twist1 expression in HCC cells, thereby suppressing metastasis and recurrence of HCC. Our study found that the difference between the incidence of metastasis in patients with positive BTBD7 expression and those with negative BTBD7 expression, as well as the positive BTBD7 expression rate of the metastatic patients and non-metastatic patients was significantly different, thus suggesting that BTBD7 may play a significant role in the process of lung cancer metastasis. Then, logistic regression analysis was performed to further confirm the relationship between BTBD7 expression and NSCLC metastasis, and the results identified that BTBD7 expression along with TNM stage were independent risk factors for NSCLC metastasis, and the risk of metastasis in patients with positive BTBD7 expression was 10.258 times as high as that in those without BTBD7 expression. Therefore, it can be inferred that BTBD7 is a critical contributor to NSCLC metastasis. However, the mechanism for BTBD7 promoting NSCLC invasion and metastasis is unknown. Based on previous studies, we hypothesized that BTBD7 regulates epithelial cell dynamics and branching morphogenesis through inducing Snail2 and suppressing E-cadherin expression, such events are associated with increased cell-to-cell adhesion, enhanced cell motility, invasive phenotypes, which is an important physiological process contributing to NSCLC invasion and metastasis $(15,16)$.

Because metastasis is closely associated with poor prognosis of lung cancer, we therefore analyzed the survival of the 93 NSCLC patients. The results showed that mean estimated survival time for the whole group of patients was (71.6 \pm 4.7$)$ months and, as expected, the patients with positive of BTBD7 expression had shorter mean survival time than those with negative BTBD7 expression. Cox regression analysis again revealed that BTBD7 expression and TNM stage were independent risk factors for survival of the patients.
In conclusion, our findings suggest that BTBD7-positive NSCLC may have a high potential for metastasis and thereby a poor prognosis, moreover, BTBD7 may serve as a predictor of metastasis and a potential therapeutic target for NSCLC. However, a full understanding of the mechanism for the activity of BTBD7 in NSCLC requires further investigations.

\section{Abbreviation}

EMT: epithelial-mesenchymal transition; BTBD7: BTB/POZ domain-containing protein 7; NSCLC: non-small-cell lung cancer; HCC: hepatocellular carcinoma; ANLT: adjacent nontumorous lung tissue; B: coefficient for the constant; SE: standard error; Sig: significance; OR: odds ratio; CI: confidence interval.

\section{Acknowledgements}

This study was supported by grants from the National Nature Science Foundation of China (No. 81102046).

\section{Competing Interests}

The authors have declared that no competing interest exists.

\section{References}

1. Bahader Y, Jazieh A. Epidemiology of lung cancer. Ann Thorac Med. 2008;3:65-67.

2. Tatard VM, Xiang C, Biegel JA, Dahmane N. ZNF238 is expressed in postmitotic brain cells and inhibits brain tumor growth. Cancer Res. 2010; 70:1236-1246.

3. Geyer R, Wee S, Anderson S, Yates J, Wolf DA. BTB/POZ domain proteins are putative substrate adaptors for cullin 3 ubiquitin ligases. Mol Cell. 2003; 12: 783-790.

4. Schmalhofer O, Brabletz S, Brabletz T. E-cadherin, beta-catenin, and ZEB1 in malignant progression of cancer. Cancer Metastasis Rev. 2009; 28:151-166.

5. Onodera T, Sakai T, Hsu JC, et al. Btbd7 regulates epithelial cell dynamics and branching morphogenesis. Science. 2010;329:562-5.

6. Awaya H, Takeshima Y, Amatya VJ, et al. Loss of expression of E-cadherin and beta-catenin is associated with progression of pulmonary adenocarcinoma. Pathol Int. 2005; 55:14-18.

7. Tao YM, Huang JL, Zeng $\mathrm{S}$, et al. BTB/POZ domain-containing protein 7 : Epithelial-mesenchymal transition promoter and prognostic biomarker of hepatocellular carcinoma? Hepatology. 2013; 57:2326-2337.

8. Kay F, Saskia G, Holger E, Walter J. Overexpression of p53 and prognosis in breast cancer. Cancer. 1993;72:3641-3647.

9. Wu F, Yang LY, Li YF, et al. Novel role for epidermal growth factor-like domain 7 in metastasis of human hepatocellular carcinoma. Hepatology. 2009; 50: 1839- 1850

10. Polo JM, Dell'Oso T, Ranuncolo SM, et al. Specific peptide interference reveals BCL6 transcriptional and oncogenic mechanisms in B-cell lymphoma cells. Nat Med. 2004; 10:1329-1335.

11. Nakayama K, Nakayama N, Davidson B, et al. A BTB/POZ protein, NAC-1, is related to tumor recurrence and is essential for tumor growth and survival. Proc Natl Acad Sci USA. 2006; 103:18739-18744.

12. Wang YP, Wang MZ, Luo YR, et al. Lentivirus-mediated shRNA interference targeting SLUG inhibits lung cancer growth and metastasis. Asian Pac J Cancer Prev. 2012; 13:4947-4951.

13. Böhm M, Totzeck B, Birchmeier W, et al. Differences of E-cadherin expression levels and patterns in primary and metastatic human lung cancer. Clin Exp Metastasis. 1994; 12:55-62.

14. Knowles LM, Gurski LA, Engel $C$, et al. Integrin $\alpha v \beta 3$ and fibronectin upregulate Slug in cancer cells to promote clot invasion and metastasis. Cancer Res. 2013; 73: 6175-6184.

15. Yang MH, Chen CL, Chau GY, et al. Comprehensive analysis of the independent effect of twist and snail in promoting metastasis of hepatocellular carcinoma. HEPATOLOGY. 2009; 50: 1464-1474.

16. phyris N, Mani SA. pIg R: frenemy of inflammation, EMT, and HCC progression. J Natl Cancer Inst. 2011; 103:1644-1645. 